\title{
Cancer chemoprevetive effects of phytochemicals by suppressing cancer metastasis
}

\begin{abstract}
Cancer development, a dynamic and long-term process, involves many complex factors with stepwise progression ultimately leading to uncontrolled spreading and growth of cancerous cells throughout the body called metastasis. The three critical steps in this process for several types of human cancer formation are initiation, promotion and progression. Invasion and metastasis are fundamental properties of malignant cancer cells. In advanced tumors, EMT (epithelial-mesenchymal transition) and mesenchymal-epithilial transition (MET) is commonly associated with acquisition of metastatic potential. Chemoprevention, a relatively new and promising strategy to prevent cancer, is defined as the use of natural dietary compounds and/or synthetic substances to block, inhibit, reverse, or retard the process of tumorigenesis. The chemopreventive effects elicited by these natural dietary compounds are believed to be the properties include antioxidative, anti-inflammatory activity, induction of phase 2 enzymes, apoptosis, and cell cycle arrest. In this review, we will discuss the phytochemicals on the potential molecular targets and signalling pathways that mediate tumour metastasis.
\end{abstract}

Keywords: chemopreventive, antioxidative, anti-inflammatory, compounds, cancer
Volume 5 Issue $6-2017$

\author{
Pei Sheng Lee, Chia Hsuan Lee, Min Hsiung \\ Pan \\ Institute of Food Science and Technology, National Taiwan \\ University, Taiwan
}

\begin{abstract}
Correspondence: Min-Hsiung Pan, Institute of Food Science and Technology, National Taiwan University, No. I, Section 4, Roosevelt Road, Taipei 10617, Taiwan, Tel (886)-2-33664I33, Fax (886)-2-3366।77I, Email mhpan@ntu.edu.tw
\end{abstract}

Received:June 16,2017| Published: August II, 2017
Abbreviations: CCL2, C-C motif ligand 2; TMP, terameprocol; DNA, deoxyribonucleic acid; ECM, extracellular matrix; MMPs, matrix metalloproteinases; MET, mesenchymal-to-epithelial transition; EMT, epithelial-mesenchymal transition

\section{Cancer Metastasis}

Cancer progression can be simplified into three stages: tumor initiation, tumor promotion and tumor metastasis. Cancer metastasis, the terminal stage of cancer development, accounts for approximately $90 \%$ of all human cancer mortalities. It is widely accepted that once cancer evolves into cancer metastasis, the speed of cancer deterioration surges and the condition will become hard to control. However, as the elusive process of cancer metastasis has been gradually deciphered, how to tackle cancer metastasis is becoming clearer. ${ }^{1}$ To figure out how phytochemicals interfere with the metastatic process, understanding the process of metastasis is very important. Metastasis can be depicted as a two-phase process: The first phase is physical translocation of cancer cells from primary tumors to distant organs and the second phase is tumor cell colonization in distant organs. From the therapeutic standpoint, understanding the mechanisms of physical translocation is critical to the prevention of metastasis for patients who are in the early stage of cancer while understanding the mechanisms leading to successful colonization may bring about effective therapies for patients with established metastases. ${ }^{1,2}$ Since colon cancer arises in epithelial tissues, this review focuses on epithelial cells. Normally, epithelial cells are bound to neighboring cells and to underlying basement membranes by adherents junctions and tight junctions which effectively immobilize epithelial cells. In order to break away from primary tumors and initiate cancer metastatic process, cancer cells will gain the ability to migrate and invade as they become more malignant. ${ }^{3}$ These two abilities enable cancer cells to decompose, move through the extracellular matrix surrounding them and enter blood and lymph vessels, two highways carrying cancer cells to distant secondary metastatic sites. As a tumor progresses, cancerous epithelial cells will go through epithelial-mesenchymal transition (EMT) to gain the ability to migrate and invade. Decreased E-cadherin, an epithelial intracellular linkage protein, is one of the hallmarks of EMT. ${ }^{4}$

EMT has been found to play a critical role in the early embryonic morphogenesis It is a reversible transition in many embryonic contexts, so it is assumed that cells which have transformed to mesenchymal phenotype may revert back to an epithelial form via mesenchymal-toepithelial transition (MET). Since EMT initiates cancer cell migration, it was supposed that inhibiting or reversing EMT process can thereby inhibit tumor metastasis. ${ }^{4}$ However, several studies have shown that disseminated cancer cells in metastatic sites are more like epithelial cells compared to cancer cells in primary tumors in a number of different types of carcinomas, including prostate cancer, breast cancer, colorectal cancer, pulmonary cancer and hepatic carcinomas . To explain this contradiction, a MET process was assumed to be involved in the formation process of metastatic tumors. ${ }^{4,5}$ Epigenetic regulation of metastasis suppressor genes promises to be a potentially important mechanism by which dietary components can moluate cancer metastasis since many phytochemicals are known to modulate gene expression. ${ }^{4,6,7}$

\section{Emt and Anoikis}

Anoikis is a type of cell death caused by cell detachment from the cell-matrix. It is a very important cancer-preventing mechanism to epithelial tissues because anoikis mechanism facilitates the elimination of unattached epithelial cells, which have been shed from the epithelium in the high-rate epithelium turnover process, to prevent these cells from attaching to inappropriate sites. Inappropriate attachment may lead to extensive neoplastic growth and cause cancers. ${ }^{5,8,9}$

Previous studies have indicated that cell detachment causes cell death, anoikis, in normal epithelial cells but not in those cancerous epithelial cells which have been through EMT because in the EMT process, cancer cells will gain the resistance to anoikis for surviving as 
they become more malignant. ${ }^{5,9}$ Two studies have strongly supported that E-cadherin-mediated cell interaction between normal epithelial cells sensitizes detached epithelial cells to anoikis. ${ }^{10}$ Previous reported that phenolic compounds and isoliquiritigenin inhibits human breast cancer metastasis through preventing anoikis resistance, migration and invasion. ${ }^{11,12}$

\section{Two Critical Barriers in the Process of Intravasation and Extravasation}

Tumor metastasis is a multi-step progressing process, including acquisition of invasive phenotype, intravasation, extravasation and colonization in distant organs. This research focused on intravasation and extravasation stages. Each of these stages has a crucial barrier. In the intravasation process, the extracellular matrix (ECM), a protein layer confining tumor cells in tissues, is a barrier to prevent tumor cells from entering blood circulation while in the extravasation process, the blood vessel wall is a barrier to inhibit tumor cells from leaving blood circulation and invading other organs. ${ }^{13}$

To enter blood circulation and move to other distant organs, tumor cells secrete matrix metalloproteinases (MMPs) to decompose ECM. MMPs compose a family of zinc and calcium-dependent proteolytic enzymes which digest various kinds of protein components of ECM, including collagen, laminin, fibronectin, vitronectin, elastin and proteoglycans. MMPs also mobilize and activate certain growth factors that have been tethered in inactive forms to the ECM or to the surface of the cells . Furthermore, previous studies have indicated that MMPs are crucial to invasion, migration, and tumorigenesis. ${ }^{13,14}$ Therefore, MMPs are main targets that should be focus on to prevent cancer cells from breaking through the ECM, a barrier in the intravasation process. ${ }^{4,15}$

After entering the blood circulation, cancer cells have to gain the ability to penetrate blood vessel walls so as to leave blood circulation system and invade other tissues or organs. In order to penetrate blood vessel walls, colon cancer cells increasingly produce $\mathrm{C}-\mathrm{C}$ motif ligand 2 (CCL2) when they become more malignant. ${ }^{16}$ CCL2, also named monocyte chemotactic protein-1 (MCP-1), is a chemokine that can recruit monocytes or other inflammation-related cells. Human MCP-1 gene is located on chromosome 17 (chr.17, q11.2). It is composed of 76 amino acids and is $13 \mathrm{kDa}$ in size. Its receptor, $\mathrm{C}-\mathrm{C}$ chemokine receptor type 2 (CCR2), is mostly expressed in vascular endothelial cells, vascular smooth muscle cells, monocytes, fibroblast and several cancer cell lines. Prior studies showed that CCL2-mediated macrophage recruitment promotes tumor growth, progression and metastasis in different cancers, including breast, ovarian and prostate cancers. ${ }^{16}$ Recent reported that terameprocol (TMP), is a naturally occurring phenolic compound found in the resin of the creosote bush, inhibited CCL2 gene expression by preventing RelA binding to DNA element.

\section{Conclusion}

Cancer metastasis is the major cause of cancer-related death Chemoprevention, a relatively new and promising strategy to prevent cancer, is defined as the use of natural dietary compounds and/or synthetic substances to block, inhibit, reverse, or retard the process of carcinogenesis. Many natural dietary compounds in fruits and vegetables have been isolated and have demonstrated healthpromoting properties. Theses phytochemicals including flavonoids, carotenoids, proanthocyanidisn, polyphenolic compounds, isothiocyanates, and terpenoids are suggested to be the active members of the natural compounds on anti-invasion and anti-metastasis. In this review, the implementation of chemoprevention of cancer metastasis by various natural dietary compounds are promising candidates for chemopreventive and chemotherapeutic cancer treatment strategies.

\section{Acknowledgements}

This study was supported by the Ministry of Science and Technology (105-2320- B-002-031-MY3 and 105-2628-B-002-003MY3).

\section{Conflicts of Interset}

None.

\section{References}

1. Pan MH, Lai CS, Wu JC, et al. Molecular mechanisms for chemoprevention of colorectal cancer by natural dietary compounds. Mol Nutr Food Res. 2011;55(1):32-45.

2. Demirkan B. The Roles of Epithelial-to-Mesenchymal Transition (EMT) and Mesenchymal-to-Epithelial Transition (MET) in Breast Cancer Bone Metastasis: Potential Targets for Prevention and Treatment. J Clin Med. 2013;2(4):264-282.

3. Brabletz T. EMT and MET in metastasis: where are the cancer stem cells? Cancer Cell. 2012;22(6):699-701.

4. Kim EK, Choi EJ, Debnath T. Role of phytochemicals in the inhibition of epithelial-mesenchymal transition in cancer metastasis. Food \& Function. 2016;7(9):3677-3685.

5. Jolly MK, Ware KE, Gilja S, et al. EMT and MET: necessary or permissive for metastasis? Mol Oncol. 2017;11(7):755-769.

6. Pan MH, Chiou YS, Chen LH, et al. Breast cancer chemoprevention by dietary natural phenolic compounds: specific epigenetic related molecular targets. Mol Nutr Food Res. 2015;59(1):21-35.

7. Meadows GG. Diet, nutrients, phytochemicals, and cancer metastasis suppressor genes. Cancer Metastasis Rev. 2012;31(3-4):441-454.

8. Yao X, Pham T, Temple B, et al. The Anoikis Effector Bit1 Inhibits EMT through Attenuation of TLE1-Mediated Repression of E-Cadherin in Lung Cancer Cells. PLoS One. 2016;11(9):e0163228.

9. Cao Z, Livas T, Kyprianou N. Anoikis and EMT: Lethal "Liaisons" during Cancer Progression. Crit Rev Oncog. 2016;21(3-4):155-168.

10. Huang RY, Wong MK, Tan TZ, et al. An EMT spectrum defines an anoikis-resistant and spheroidogenic intermediate mesenchymal state that is sensitive to e-cadherin restoration by a src-kinase inhibitor, saracatinib (AZD0530). Cell Death Dis. 2014;4:e915.

11. Tanagornmeatar K, Chaotham C, Sritularak B, et al. Cytotoxic and anti-metastatic activities of phenolic compounds from Dendrobium ellipsophyllum. Anticancer Res. 2014;34(11):6573-6579.

12. Zheng H, Li Y, Wang Y, et al. Downregulation of COX-2 and CYP 4A signaling by isoliquiritigenin inhibits human breast cancer metastasis through preventing anoikis resistance, migration and invasion. Toxicol Appl Pharmacol. 2014;280(1):10-20.

13. Weng CJ, Yen GC. Chemopreventive effects of dietary phytochemicals against cancer invasion and metastasis: phenolic acids, monophenol, polyphenol, and their derivatives. Cancer Treat Rev. 2012;38(1):76-87.

14. Pan MH, Chiou YS, Wang YJ, et al. Multistage carcinogenesis process as molecular targets in cancer chemoprevention by epicatechin-3gallate. Food Funct. 2011;2(2):101-110.

15. Shu L, Cheung KL, Khor TO, et al. Phytochemicals: cancer chemoprevention and suppression of tumor onset and metastasis. Cancer Metastasis Rev. 2010;29(3):483-502.

16. Chun E, Lavoie S, Michaud M, et al. CCL2 Promotes Colorectal Carcinogenesis by Enhancing Polymorphonuclear Myeloid-Derived Suppressor Cell Population and Function. Cell Rep. 2015;12(2):244-257. 